\title{
Homotopy Perturbation Method for Fractional Gas Dynamics Equation Using Sumudu Transform
}

\author{
Jagdev Singh, ${ }^{1}$ Devendra Kumar, ${ }^{2}$ and A. Kılıçman ${ }^{3}$ \\ ${ }^{1}$ Department of Mathematics, Jagannath University, Rampura, Chaksu, Jaipur, Rajasthan 303901, India \\ ${ }^{2}$ Department of Mathematics, Jagannath Gupta Institute of Engineering \& Technology, Jaipur, Rajasthan 302022, India \\ ${ }^{3}$ Department of Mathematics and Institute for Mathematical Research, University Putra Malaysia (UPM), \\ 43400 Serdang, Selangor, Malaysia
}

Correspondence should be addressed to A. Kılıçman; akilicman@putra.upm.edu.my

Received 12 September 2012; Revised 19 November 2012; Accepted 6 December 2012

Academic Editor: Lan $\mathrm{Xu}$

Copyright (C) 2013 Jagdev Singh et al. This is an open access article distributed under the Creative Commons Attribution License, which permits unrestricted use, distribution, and reproduction in any medium, provided the original work is properly cited.

\begin{abstract}
A user friendly algorithm based on new homotopy perturbation Sumudu transform method (HPSTM) is proposed to solve nonlinear fractional gas dynamics equation. The fractional derivative is considered in the Caputo sense. Further, the same problem is solved by Adomian decomposition method (ADM). The results obtained by the two methods are in agreement and hence this technique may be considered an alternative and efficient method for finding approximate solutions of both linear and nonlinear fractional differential equations. The HPSTM is a combined form of Sumudu transform, homotopy perturbation method, and He's polynomials. The nonlinear terms can be easily handled by the use of He's polynomials. The numerical solutions obtained by the proposed method show that the approach is easy to implement and computationally very attractive.
\end{abstract}

\section{Introduction}

Fractional calculus is a field of applied mathematics that deals with derivatives and integrals of arbitrary orders. During the last decade, fractional calculus has found applications in numerous seemingly diverse fields of science and engineering. Fractional differential equations are increasingly used to model problems in fluid mechanics, acoustics, biology, electromagnetism, diffusion, signal processing, and many other physical processes [1-19].

There exists a wide class of literature dealing with the problems of approximate solutions to fractional differential equations with various different methodologies, called perturbation methods. The perturbation methods have some limitations; for example, the approximate solution involves series of small parameters which poses difficulty since the majority of nonlinear problems have no small parameters at all. Although appropriate choices of small parameters sometimes lead to ideal solution, in most of the cases unsuitable choices lead to serious effects in the solutions. Therefore, an analytical method is welcome which does not require a small parameter in the equation modeling the phenomenon.
Recently, there is a very comprehensive literature review in some new asymptotic methods for the search for the solitary solutions of nonlinear differential equations, nonlinear differential-difference equations, and nonlinear fractional differential equations; see [20]. The homotopy perturbation method (HPM) was first introduced by He [21]. The HPM was also studied by many authors to handle linear and nonlinear equations arising in various scientific and technological fields [22-32]. The Adomian decomposition method (ADM) [33] and variational iteration method (VIM) [34] have also been applied to study the various physical problems.

In a recent paper, Singh et al. [35] have paid attention to study the solutions of linear and nonlinear partial differential equations by using the homotopy perturbation Sumudu transform method (HPSTM). The HPSTM is a combination of Sumudu transform, HPM, and He's polynomials and is mainly due to Ghorbani and Saberi-Nadjafi [36] and Ghorbani [37].

In this paper, we consider the following nonlinear timefractional gas dynamics equation of the form

$$
D_{t}^{\alpha} U+\frac{1}{2}\left(U^{2}\right)_{x}-U(1-U)=0, \quad t>0,0<\alpha \leq 1,
$$


with the initial condition

$$
U(x, 0)=e^{-x},
$$

where $\alpha$ is a parameter describing the order of the fractional derivative. The function $U(x, t)$ is the probability density function, $t$ is the time, and $x$ is the spatial coordinate. The derivative is understood in the Caputo sense. The general response expression contains a parameter describing the order of the fractional derivative that can be varied to obtain various responses. In the case of $\alpha=1$ the fractional gas dynamics equation reduces to the classical gas dynamics equation. The gas dynamics equations are based on the physical laws of conservation, namely, the laws of conservation of mass, conservation of momentum, conservation of energy, and so forth. The nonlinear fractional gas dynamics has been studied previously by Das and Kumar [38].

Further, we apply the HPSTM and ADM to solve the nonlinear time-fractional gas dynamics equation. The objective of the present paper is to extend the application of the HPSTM to obtain analytic and approximate solutions to the time-fractional gas dynamics equation. The advantage of the HPSTM is its capability of combining two powerful methods for obtaining exact and approximate analytical solutions for nonlinear equations. It provides the solutions in terms of convergent series with easily computable components in a direct way without using linearization, perturbation, or restrictive assumptions. It is worth mentioning that the HPSTM is capable of reducing the volume of the computational work as compared to the classical methods while still maintaining the high accuracy of the numerical result; the size reduction amounts to an improvement of the performance of the approach.

\section{Sumudu Transform}

In the early 90's, Watugala [39] introduced a new integral transform, named the Sumudu transform and applied it to the solution of ordinary differential equation in control engineering problems. The Sumudu transform, is defined over the set of functions

$$
\begin{aligned}
A=\{ & f(t)\left|\exists M, \tau_{1}, \tau_{2}>0,\right| f(t) \mid \\
& \left.<M e^{|t| / \tau_{j}}, \text { if } t \in(-1)^{j} \times[0, \infty)\right\}
\end{aligned}
$$

by the following formula:

$$
\begin{aligned}
\bar{f}(u) & =S[f(t)] \\
& =\int_{0}^{\infty} f(u t) e^{-t} d t, \quad u \in\left(-\tau_{1}, \tau_{2}\right) .
\end{aligned}
$$

Some of the properties were established by Weerakoon in [40, 41]. In [42], by Aşiru, further fundamental properties of this transform were also established. Similarly, this transform was applied to the one-dimensional neutron transport equation in [43] by Kadem. In fact it was shown that there is a strong relationship between Sumudu and other integral transforms; see Kılıçman et al. [44]. In particular the relation between Sumudu transform and Laplace transforms was proved in K1lıçman and Gadain [45].

Further, in Eltayeb et al. [46], the Sumudu transform was extended to the distributions and some of their properties were also studied in Kılıçman and Eltayeb [47]. Recently, this transform is applied to solve the system of differential equations; see Kılıçman et al. in [48].

Note that a very interesting fact about Sumudu transform is that the original function and its Sumudu transform have the same Taylor coefficients except the factor $n$; see Zhang [49]. Thus if $f(t)=\sum_{n=0}^{\infty} a_{n} t^{n}$ then $F(u)=\sum_{n=0}^{\infty} n ! a_{n} u^{n}$; see Kilıçman et al. [44]. Similarly, the Sumudu transform sends combinations, $C(m, n)$, into permutations, $P(m, n)$, and hence it will be useful in the discrete systems.

\section{Basic Definitions of Fractional Calculus}

In this section, we mention the following basic definitions of fractional calculus which are used further in the present paper.

Definition 1. The Riemann-Liouville fractional integral operator of order $\alpha>0$, of a function $f(t) \in C_{\mu}$, and $\mu \geq-1$ is defined as [5]

$$
\begin{gathered}
J^{\alpha} f(t)=\frac{1}{\Gamma(\alpha)} \int_{0}^{t}(t-\tau)^{\alpha-1} f(\tau) d \tau, \quad(\alpha>0), \\
J^{0} f(t)=f(t) .
\end{gathered}
$$

For the Riemann-Liouville fractional integral, we have

$$
J^{\alpha} t^{\gamma}=\frac{\Gamma(\gamma+1)}{\Gamma(\gamma+\alpha+1)} t^{\alpha+\gamma}
$$

Definition 2. The fractional derivative of $f(t)$ in the Caputo sense is defined as [10]

$$
\begin{aligned}
D_{t}^{\alpha} f(t) & =J^{m-\alpha} D^{n} f(t) \\
& =\frac{1}{\Gamma(n-\alpha)} \int_{0}^{t}(t-\tau)^{m-\alpha-1} f^{(m)}(\tau) d \tau,
\end{aligned}
$$

for $m-1<\alpha \leq m, m \in N, t>0$.

For the Riemann-Liouville fractional integral and the Caputo fractional derivative, we have the following relation:

$$
J_{t}^{\alpha} D_{t}^{\alpha} f(t)=f(t)-\sum_{k=0}^{m-1} f^{(k)}(0+) \frac{t^{k}}{k !}
$$

Definition 3. The Sumudu transform of the Caputo fractional derivative is defined as follows [50]:

$$
\begin{aligned}
S\left[D_{t}^{\alpha} f(t)\right]= & u^{-\alpha} S[f(t)] \\
& -\sum_{k=0}^{m-1} u^{-\alpha+k} f^{(k)}(0+), \quad(m-1<\alpha \leq m) .
\end{aligned}
$$




\section{Solution by Homotopy Perturbation Sumudu Transform Method (HPSTM)}

4.1. Basic Idea of HPSTM. To illustrate the basic idea of this method, we consider a general fractional nonlinear nonhomogeneous partial differential equation with the initial condition of the form

$$
\begin{gathered}
D_{t}^{\alpha} U(x, t)+R U(x, t)+N U(x, t)=g(x, t), \\
U(x, 0)=f(x),
\end{gathered}
$$

where $D_{t}^{\alpha} U(x, t)$ is the Caputo fractional derivative of the function $U(x, t), R$ is the linear differential operator, $N$ represents the general nonlinear differential operator, and $g(x, t)$ is the source term.

Applying the Sumudu transform (denoted in this paper by $S$ ) on both sides of (11), we get

$$
S\left[D_{t}^{\alpha} U(x, t)\right]+S[R U(x, t)]+S[N U(x, t)]=S[g(x, t)] .
$$

Using the property of the Sumudu transform, we have

$$
\begin{aligned}
S[U(x, t)]= & f(x)+u^{\alpha} S[g(x, t)] \\
& -u^{\alpha} S[R U(x, t)+N U(x, t)] .
\end{aligned}
$$

Operating with the Sumudu inverse on both sides of (14) gives

$$
U(x, t)=G(x, t)-S^{-1}\left[u^{\alpha} S[R U(x, t)+N U(x, t)]\right],
$$

where $G(x, t)$ represents the term arising from the source term and the prescribed initial conditions. Now we apply the HPM:

$$
U(x, t)=\sum_{n=0}^{\infty} p^{n} U_{n}(x, t),
$$

and the nonlinear term can be decomposed as

$$
N U(x, t)=\sum_{n=0}^{\infty} p^{n} H_{n}(U),
$$

for some He's polynomials $H_{n}(U)$ [37] that are given by

$$
\begin{aligned}
& H_{n}\left(U_{0}, U_{1}, \ldots, U_{n}\right) \\
& \quad=\frac{1}{n !} \frac{\partial^{n}}{\partial p^{n}}\left[N\left(\sum_{i=0}^{\infty} p^{i} U_{i}\right)\right]_{p=0}, \quad n=0,1,2, \ldots
\end{aligned}
$$

Substituting (16) and (17) in (15), we get

$$
\begin{aligned}
& \sum_{n=0}^{\infty} p^{n} U_{n}(x, t) \\
& =G(x, t) \\
& \quad-p\left(S^{-1}\left[u^{\alpha} S\left[R \sum_{n=0}^{\infty} p^{n} U_{n}(x, t)+\sum_{n=0}^{\infty} p^{n} H_{n}(U)\right]\right]\right),
\end{aligned}
$$

which is the coupling of the Sumudu transform and the HPM using He's polynomials. Comparing the coefficients of like powers of $p$, the following approximations are obtained:

$$
\begin{gathered}
p^{0}: U_{0}(x, t)=G(x, t), \\
p^{1}: U_{1}(x, t)=-S^{-1}\left[u^{\alpha} S\left[R U_{0}(x, t)+H_{0}(U)\right]\right], \\
p^{2}: U_{2}(x, t)=-S^{-1}\left[u^{\alpha} S\left[R U_{1}(x, t)+H_{1}(U)\right]\right], \\
p^{3}: U_{3}(x, t)=-S^{-1}\left[u^{\alpha} S\left[R U_{2}(x, t)+H_{2}(U)\right]\right],
\end{gathered}
$$

Proceeding in this same manner, the rest of the components $U_{n}(x, t)$ can be completely obtained and the series solution is thus entirely determined. Finally, we approximate the analytical solution $U(x, t)$ by truncated series:

$$
U(x, t)=\lim _{N \rightarrow \infty} \sum_{n=0}^{N} U_{n}(x, t) .
$$

The above series solutions generally converge very rapidly.

4.2. Solution of the Problem. Consider the following nonlinear time-fractional gas dynamics equation:

$$
D_{t}^{\alpha} U+\frac{1}{2}\left(U^{2}\right)_{x}-U(1-U)=0, \quad 0<\alpha \leq 1,
$$

with the initial condition

$$
U(x, 0)=e^{-x} .
$$

Applying the Sumudu transform on both sides of (22), subject to the initial condition (23), we have

$$
S[U(x, t)]=e^{-x}-u^{\alpha} S\left[\frac{1}{2}\left(U^{2}\right)_{x}-U(1-U)\right] .
$$

The inverse Sumudu transform implies that

$$
U(x, t)=e^{-x}-S^{-1}\left[u^{\alpha} S\left[\frac{1}{2}\left(U^{2}\right)_{x}-U(1-U)\right]\right]
$$

Now applying the HPM, we get

$$
\begin{aligned}
& \sum_{n=0}^{\infty} p^{n} U_{n}(x, t) \\
&=e^{-x}-p\left(S ^ { - 1 } \left[u ^ { \alpha } S \left[\frac{1}{2}\left(\sum_{n=0}^{\infty} p^{n} H_{n}(U)\right)\right.\right.\right. \\
&-\left(\sum_{n=0}^{\infty} p^{n} U_{n}(x, t)\right) \\
&\left.\left.\left.+\left(\sum_{n=0}^{\infty} p^{n} H_{n}^{\prime}(U)\right)\right]\right]\right)
\end{aligned}
$$


where $H_{n}(U)$ and $H_{n}^{\prime}(U)$ are He's polynomials [37] that represent the nonlinear terms. So, the He's polynomials are given by

$$
\sum_{n=0}^{\infty} p^{n} H_{n}(U)=\left(U^{2}\right)_{x}
$$

The first few components of He's polynomials are given by

$$
\begin{gathered}
H_{0}(U)=\left(U_{0}^{2}\right)_{x}, \\
H_{1}(U)=2\left(U_{0} U_{1}\right)_{x} \\
H_{1}(U)=\left(U_{1}^{2}+2 U_{0} U_{2}\right)_{x},
\end{gathered}
$$

and for $H_{n}^{\prime}(U)$, we find that

$$
\begin{gathered}
\sum_{n=0}^{\infty} p^{n} H_{n}^{\prime}(U)=U^{2}, \\
H_{0}^{\prime}(U)=U_{0}^{2}, \\
H_{1}^{\prime}(U)=2 U_{0} U_{1}, \\
H_{2}^{\prime}(U)=U_{1}^{2}+2 U_{0} U_{2},
\end{gathered}
$$

Comparing the coefficients of like powers of $p$, we have

$$
\begin{aligned}
& p^{0}: U_{0}(x, t)=e^{-x}, \\
p^{1}: U_{1}(x, t)= & -S^{-1}\left[u^{\alpha} S\left[\frac{1}{2} H_{0}(U)-U_{0}+H_{0}^{\prime}(U)\right]\right] \\
= & e^{-x} \frac{t^{\alpha}}{\Gamma(\alpha+1)}, \\
p^{2}: U_{2}(x, t)= & -S^{-1}\left[u^{\alpha} S\left[\frac{1}{2} H_{1}(U)-U_{1}+H_{1}^{\prime}(U)\right]\right] \\
= & e^{-x} \frac{t^{2 \alpha}}{\Gamma(2 \alpha+1)}, \\
p^{3}: U_{3}(x, t)= & -S^{-1}\left[u^{\alpha} S\left[\frac{1}{2} H_{2}(U)-U_{2}+H_{2}^{\prime}(U)\right]\right] \\
= & e^{-x} \frac{t^{3 \alpha}}{\Gamma(3 \alpha+1)},
\end{aligned}
$$

Therefore, the series solution is

$U(x, t)$

$$
=e^{-x}\left[1+\frac{t^{\alpha}}{\Gamma(\alpha+1)}+\frac{t^{2 \alpha}}{\Gamma(2 \alpha+1)}+\frac{t^{3 \alpha}}{\Gamma(3 \alpha+1)}+\cdots\right] .
$$

Setting $\alpha=1$ in (31), we reproduce the solution of the problem as follows:

$$
U(x, t)=e^{-x}\left(1+t+\frac{t^{2}}{2 !}+\frac{t^{3}}{3 !}+\cdots\right) .
$$

This solution is equivalent to the exact solution in closed form:

$$
U(x, t)=e^{t-x} .
$$

Now, we calculate numerical results of the probability density function $U(x, t)$ for different time-fractional Brownian motions $\alpha=1 / 3,2 / 3,1$ and for various values of $t$ and $x$. The numerical results for the approximate solution (31) obtained by using HPSTM and the exact solution (33) for various values of $t, x$, and $\alpha$ are shown in Figures 1(a)-1(d) and those for different values of $t$ and $\alpha$ at $x=1$ are depicted in Figure 2 .

It is observed from Figures 1 and 2 that $U(x, t)$ increases with the increase in $t$ and decreases with the increase in $\alpha$. Figures $1(\mathrm{c})$ and $1(\mathrm{~d})$ clearly show that, when $\alpha=1$, the approximate solution (31) obtained by the present method is very near to the exact solution. It is to be noted that only the third-order term of the HPSTM was used in evaluating the approximate solutions for Figures 1 and 2. It is evident that the efficiency of the present method can be dramatically enhanced by computing further terms of $U(x, t)$ when the HPSTM is used.

\section{Solution by Adomian Decomposition Method (ADM)}

5.1. Basic Idea of ADM. To illustrate the basic idea of ADM [51, 52], we consider a general fractional nonlinear nonhomogeneous partial differential equation with the initial condition of the form

$$
D_{t}^{\alpha} U(x, t)+R U(x, t)+N U(x, t)=g(x, t),
$$

where $D_{t}^{\alpha} U(x, t)$ is the Caputo fractional derivative of the function $U(x, t), R$ is the linear differential operator, $N$ represents the general nonlinear differential operator, and $g(x, t)$ is the source term.

Applying the operator $J_{t}^{\alpha}$ on both sides of (34) and using result (9), we have

$$
\begin{aligned}
U(x, t)= & \sum_{k=0}^{m-1}\left(\frac{\partial^{k} U}{\partial t^{k}}\right)_{t=0} \frac{t^{k}}{k !} \\
& +J_{t}^{\alpha} g(x, t)-J_{t}^{\alpha}[R U(x, t)+N U(x, t)] .
\end{aligned}
$$

Next, we decompose the unknown function $U(x, t)$ into sum of an infinite number of components given by the decomposition series

$$
U=\sum_{n=0}^{\infty} U_{n}
$$

and the nonlinear term can be decomposed as

$$
N U=\sum_{n=0}^{\infty} A_{n},
$$




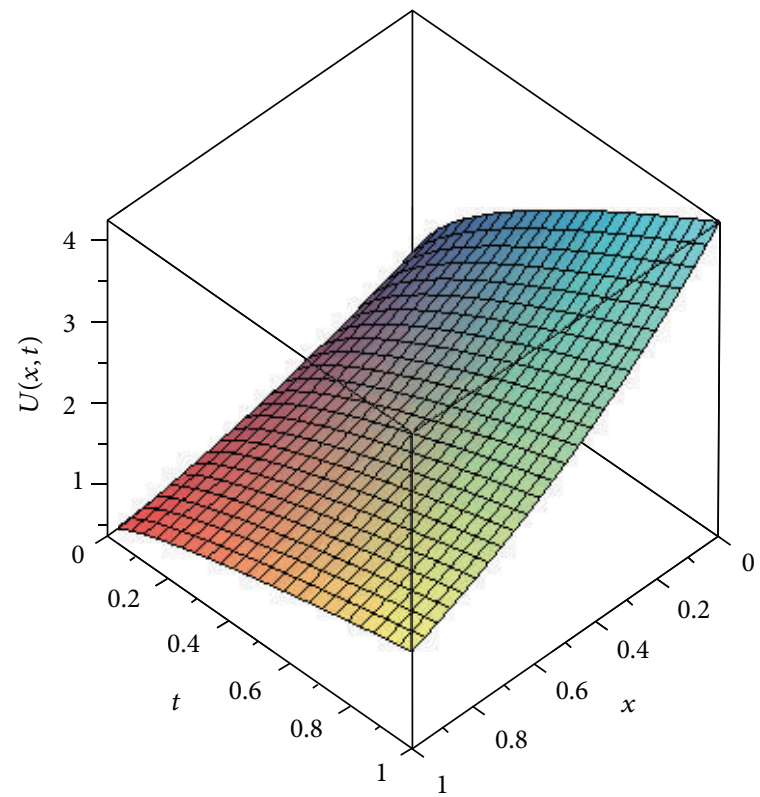

(a)

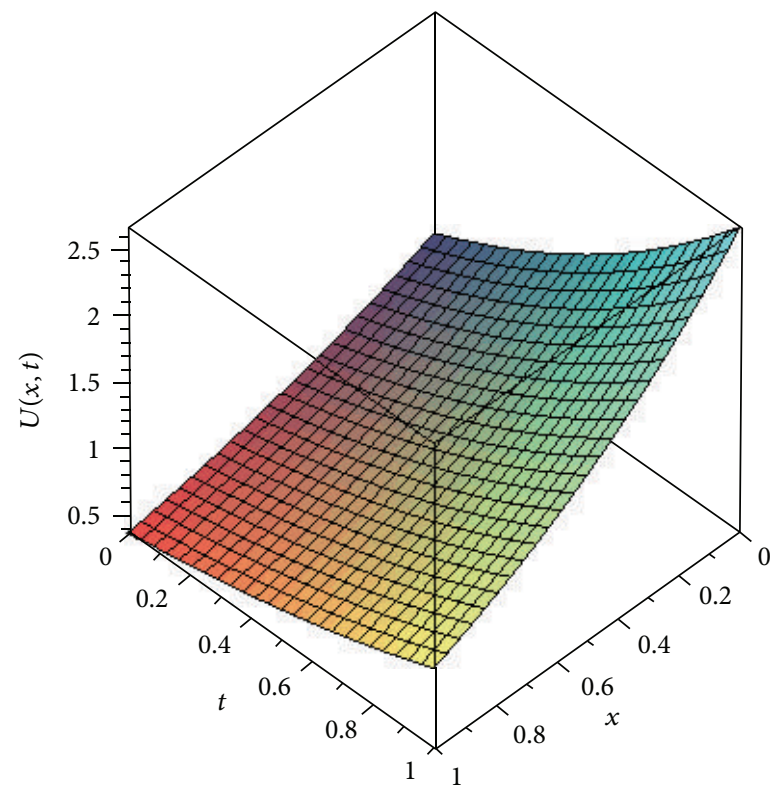

(c)

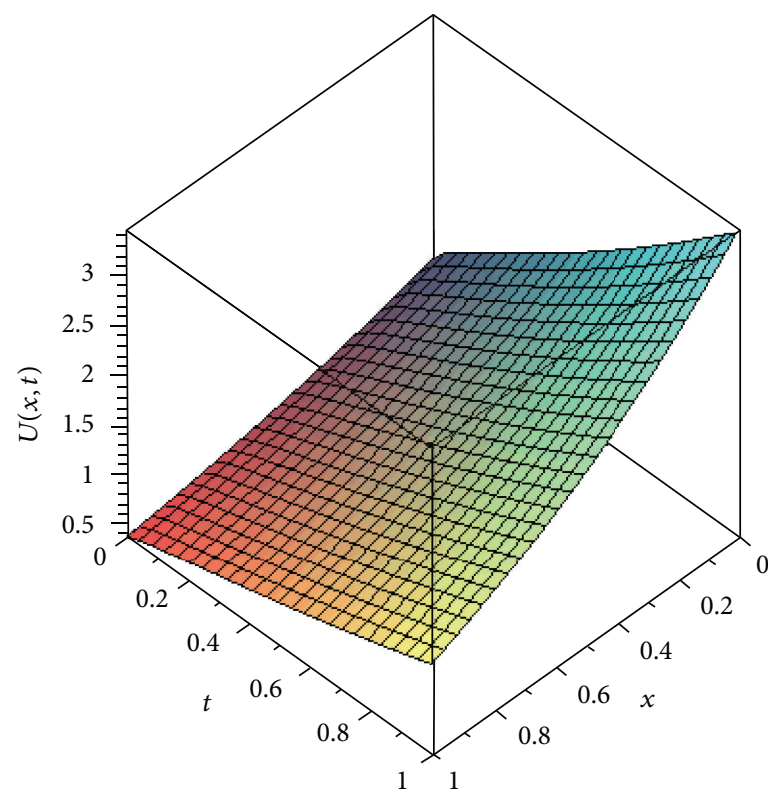

(b)

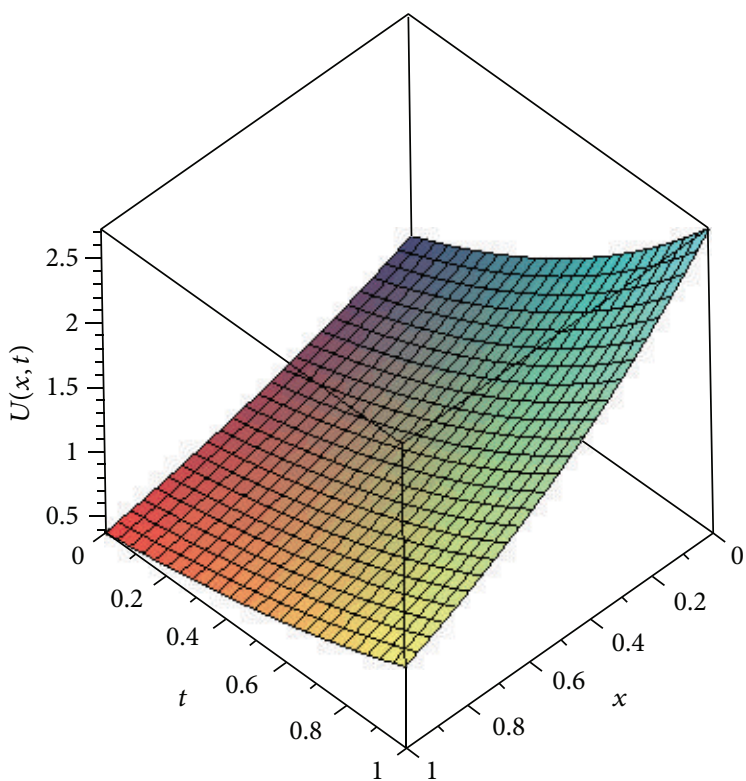

(d)

Figure 1: The behaviour of the $U(x, t)$ w.r.t. $x$ and $t$ are obtained when (a) $\alpha=1 / 3$, (b) $\alpha=2 / 3$, (c) $\alpha=1$, and (d) exact solution.

where $A_{n}$ are Adomian polynomials that are given by

$$
A_{n}=\frac{1}{n !}\left[\frac{d^{n}}{d \lambda^{n}} N\left(\sum_{i=0}^{n} \lambda^{i} U_{i}\right)\right]_{\lambda=0}, \quad n=0,1,2, \ldots
$$

The components $U_{0}, U_{1}, U_{2}, \ldots$ are determined recursively by substituting (36) and (37) into (34) leading to

$$
\begin{aligned}
\sum_{n=0}^{\infty} U_{n}= & \sum_{k=0}^{m-1}\left(\frac{\partial^{k} U}{\partial t^{k}}\right)_{t=0} \frac{t^{k}}{k !} \\
& +J_{t}^{\alpha} g(x, t)-J_{t}^{\alpha}\left[R\left(\sum_{n=0}^{\infty} U_{n}\right)+\sum_{n=0}^{\infty} A_{n}\right] .
\end{aligned}
$$

This can be written as

$$
\begin{aligned}
U_{0}+U_{1}+U_{2}+\cdots= & \sum_{k=0}^{m-1}\left(\frac{\partial^{k} U}{\partial t^{k}}\right)_{t=0} \frac{t^{k}}{k !}+J_{t}^{\alpha} g(x, t) \\
& -J_{t}^{\alpha}\left[R\left(U_{0}+U_{1}+U_{2}+\cdots\right)\right. \\
& \left.+\left(A_{0}+A_{1}+A_{2}+\cdots\right)\right] .
\end{aligned}
$$

Adomian method uses the formal recursive relations as

$$
\begin{aligned}
& U_{0}=\sum_{k=0}^{m-1}\left(\frac{\partial^{k} U}{\partial t^{k}}\right)_{t=0} \frac{t^{k}}{k !}+J_{t}^{\alpha} g(x, t), \\
& U_{n+1}=-J_{t}^{\alpha}\left[R\left(U_{n}\right)+A_{n}\right], \quad n \geq 0 .
\end{aligned}
$$


TABLE 1: Comparison study between HPSTM, ADM, and the exact solution when $\alpha=1$.

\begin{tabular}{lcccc}
\hline$x$ & $t$ & HPSTM & ADM & Exact solution \\
\hline 0 & 0.1 & 1.221333333 & 1.221333333 & 1.221402758 \\
0.2 & 0.1 & 0.9999431595 & 0.9999431595 & 1.000000000 \\
0.4 & 0.1 & 0.8186842160 & 0.8186842160 & 0.8187307531 \\
0.6 & 0.1 & 0.6702819447 & 0.6702819447 & 0.6703200460 \\
0.8 & 0.1 & 0.5487804413 & 0.5487804413 & 0.5488116361 \\
1.0 & 0.1 & 0.4493037263 & 0.4493037263 & 0.4493289641 \\
\hline
\end{tabular}

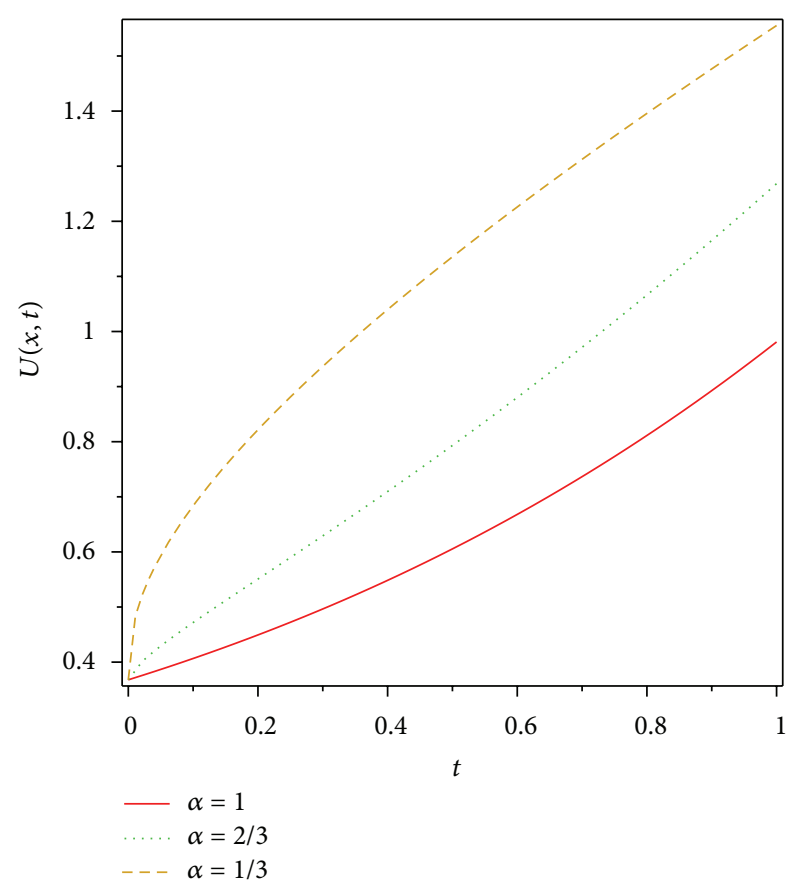

Figure 2: Plots of $U(x, t)$ versus $t$ at $x=1$ for different values of $\alpha$.

5.2. Solution of the Problem. Consider the following nonlinear time-fractional gas dynamics equation:

$$
D_{t}^{\alpha} U+\frac{1}{2}\left(U^{2}\right)_{x}-U(1-U)=0, \quad 0<\alpha \leq 1,
$$

with the initial condition

$$
U(x, 0)=e^{-x} .
$$

Applying the operator $J_{t}^{\alpha}$ on both sides of (42) and using result (9), we have

$$
U=\sum_{k=0}^{1-1} \frac{t^{k}}{k !}\left[D_{t}^{k} U\right]_{t=0}-J_{t}^{\alpha}\left[\frac{1}{2}\left(U^{2}\right)_{x}-U+U^{2}\right] .
$$

This gives the following recursive relations using (41):

$$
\begin{gathered}
U_{0}=\sum_{k=0}^{0} \frac{t^{k}}{k !}\left[D_{t}^{k} U\right]_{t=0}, \\
U_{n+1}=-J_{t}^{\alpha}\left[A_{n}-U_{n}\right], \quad n=0,1,2, \ldots,
\end{gathered}
$$

where

$$
\begin{array}{r}
A_{n}=\frac{1}{n !}\left[\left(\frac{1}{2} \frac{\partial}{\partial x}+1\right) \frac{d^{n}}{d \lambda^{n}}\left(\sum_{i=0}^{n} \lambda^{i} U_{i}\right)^{2}\right]_{\lambda=0}, \\
n=0,1,2, \ldots
\end{array}
$$

which using the results (7), (5), and (43) gives

$$
\begin{gathered}
U_{0}(x, t)=e^{-x}, \\
A_{0}=0 \\
U_{1}(x, t)=e^{-x} \frac{t^{\alpha}}{\Gamma(\alpha+1)}, \\
A_{1}=0, \\
U_{2}(x, t)=e^{-x} \frac{t^{2 \alpha}}{\Gamma(2 \alpha+1)}, \\
A_{2}=0, \\
U_{3}(x, t)=e^{-x} \frac{t^{3 \alpha}}{\Gamma(3 \alpha+1)},
\end{gathered}
$$

Therefore, the decomposition series solution is

$$
\begin{aligned}
& U(x, t) \\
& \quad=e^{-x}\left[1+\frac{t^{\alpha}}{\Gamma(\alpha+1)}+\frac{t^{2 \alpha}}{\Gamma(2 \alpha+1)}+\frac{t^{3 \alpha}}{\Gamma(3 \alpha+1)}+\cdots\right]
\end{aligned}
$$

which is the same solution as obtained by using HPSTM.

From Table 1, it is observed that the values of the approximate solution at different grid points obtained by the HPSTM and ADM are close to the values of the exact solution with high accuracy at the third-term approximation. It can also be noted that the accuracy increases as the order of approximation increases.

The comparison between the third iteration solution of the HPSTM and the second iteration solution of the ADM is given in Figure 3.

It is observed that for $x=1$ and $\alpha=1$, there is a good agreement between the two methods. 


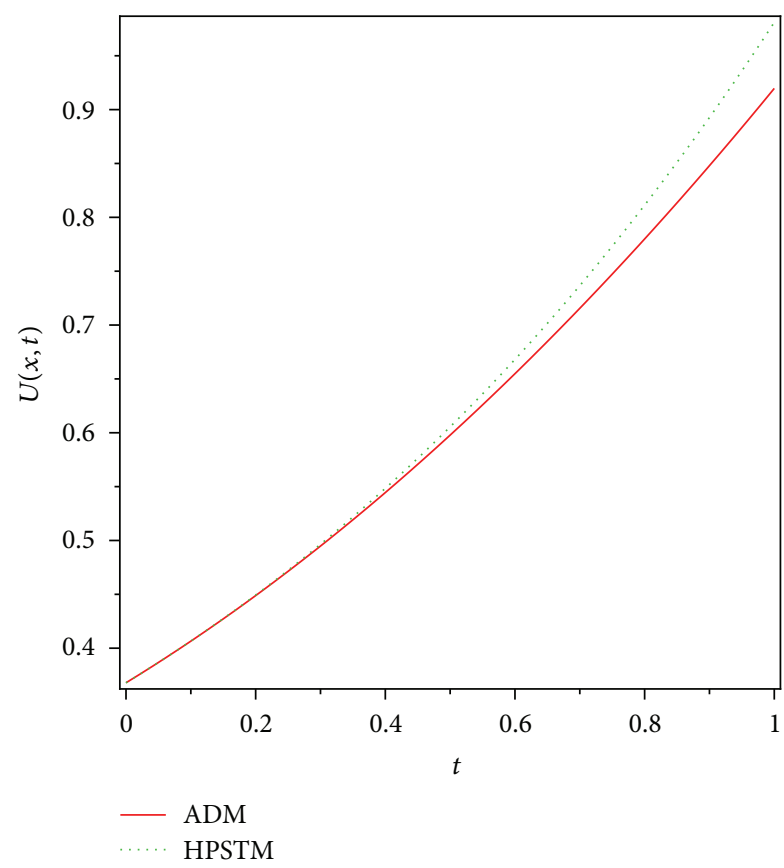

Figure 3: Comparison of the HPSTM and the ADM when $x=1$ and $\alpha=1$.

\section{Conclusions}

In this paper, the homotopy perturbation Sumudu transform method (HPSTM) and the Adomian decomposition method (ADM) are successfully applied for solving nonlinear timefractional gas dynamics equation. The numerical solutions show that there is a good agreement between the two methods. Therefore, these two methods are very powerful and efficient techniques for solving different kinds of linear and nonlinear fractional differential equations arising in different fields of science and engineering. However, the HPSTM has an advantage over the ADM which is that it solves the nonlinear problems without using Adomian polynomials. In conclusion, the HPSTM and the ADM may be considered as a nice refinement in existing numerical techniques and might find the wide applications.

\section{Acknowledgment}

The authors are grateful to the referees for their invaluable suggestions and comments for the improvement of the paper.

\section{References}

[1] H. Beyer and S. Kempfle, "Definition of physically consistent damping laws with fractional derivatives," Zeitschrift für Angewandte Mathematik und Mechanik, vol. 75, no. 8, pp. 623-635, 1995.

[2] J. H. He, "Some applications of nonlinear fractional differential equations and their approximations," Bulletin of Science, Technology \& Society, vol. 15, no. 2, pp. 86-90, 1999.

[3] J.-H. He, "Approximate analytical solution for seepage flow with fractional derivatives in porous media," Computer Methods in
Applied Mechanics and Engineering, vol. 167, no. 1-2, pp. 57-68, 1998.

[4] R. Hilfer, "Fractional time evolution," in Applications of Fractional Calculus in Physics, pp. 87-130, World Scientific, River Edge, NJ, USA, 2000.

[5] I. Podlubny, Fractional Differential Equations: An Introduction to Fractional Derivatives, Fractional Differential Equations, to Methods of Their Solution and some of Their Applications, vol. 198 of Mathematics in Science and Engineering, Academic Press, San Diego, Calif, USA, 1999.

[6] F. Mainardi, Y. Luchko, and G. Pagnini, “The fundamental solution of the space-time fractional diffusion equation," Fractional Calculus \& Applied Analysis, vol. 4, no. 2, pp. 153-192, 2001.

[7] S. Z. Rida, A. M. A. El-Sayed, and A. A. M. Arafa, "On the solutions of time-fractional reaction-diffusion equations," Communications in Nonlinear Science and Numerical Simulation, vol. 15, no. 12, pp. 3847-3854, 2010.

[8] A. Yıldırım, "He's homotopy perturbation method for solving the space- and time-fractional telegraph equations," International Journal of Computer Mathematics, vol. 87, no. 13, pp. 2998-3006, 2010.

[9] L. Debnath, "Fractional integral and fractional differential equations in fluid mechanics," Fractional Calculus \& Applied Analysis, vol. 6, no. 2, pp. 119-155, 2003.

[10] M. Caputo, Elasticita E Dissipazione, Zani-Chelli, Bologna, Italy, 1969.

[11] K. S. Miller and B. Ross, An Introduction to the Fractional Calculus and Fractional Differential Equations, John Wiley \& Sons, New York, NY, USA, 1993.

[12] K. B. Oldham and J. Spanier, The Fractional Calculus: Theory and Applications of Differentiation and Integration to Arbitrary Order, With an Annotated Chronological Bibliography by Bertram Ross, Mathematics in Science and Engineering, vol. 111, Academic Press, New York, NY, USA, 1974.

[13] A. A. Kilbas, H. M. Srivastava, and J. J. Trujillo, Theory and Applications of Fractional Differential Equations, vol. 204 of North-Holland Mathematics Studies, Elsevier Science B.V., Amsterdam, The Netherlands, 2006.

[14] X. J. Yang, Advanced Local Fractional Calculus and Its Applications, World Science Publisher, New York, NY, USA, 2012.

[15] X. J. Yang, "Local fractional integral transforms," Progress in Nonlinear Science, vol. 4, pp. 1-225, 2011.

[16] X. J. Yang, Local Fractional Functional Analysis and Its Applications, Asian Academic, Hong Kong, China, 2011.

[17] X. J. Yang, "Heat transfer in discontinuous media," Advances in Mechanical Engineering and Its Applications, vol. 1, no. 3, pp. 4753, 2012.

[18] X. J. Yang, "Local fractional partial differential equations with fractal boundary problems," Advances in Computational Mathematics and Its Applications, vol. 1, no. 1, pp. 60-63, 2012.

[19] D. Q. Zeng and Y. M. Qin, "The Laplace-Adomian-Pade technique for the seepage flows with the Riemann-Liouville derivatives," Communications in Fractional Calculus, vol. 3, no. 1, pp. 26-29, 2012.

[20] J. H. He, "Asymptotic methods for solitary solutions and compactons," Abstract and Applied Analysis, vol. 2012, Article ID 916793, 130 pages, 2012.

[21] J.-H. He, "Homotopy perturbation technique," Computer Methods in Applied Mechanics and Engineering, vol. 178, no. 3-4, pp. 257-262, 1999. 
[22] J.-H. He, "Homotopy perturbation method: a new nonlinear analytical technique," Applied Mathematics and Computation, vol. 135, no. 1, pp. 73-79, 2003.

[23] J.-H. He, "New interpretation of homotopy perturbation method. Addendum: 'some asymptotic methods for strongly nonlinear equations,' International Journal of Modern Physics $B$, vol. 20, no. 18, pp. 2561-2568, 2006.

[24] D. D. Ganji, “The application of He's homotopy perturbation method to nonlinear equations arising in heat transfer," Physics Letters A, vol. 355, no. 4-5, pp. 337-341, 2006.

[25] A. Yildirim, "An algorithm for solving the fractional nonlinear Schrödinger equation by means of the homotopy perturbation method," International Journal of Nonlinear Sciences and Numerical Simulation, vol. 10, no. 4, pp. 445-450, 2009.

[26] D. D. Ganji and M. Rafei, "Solitary wave solutions for a generalized Hirota-Satsuma coupled KdV equation by homotopy perturbation method," Physics Letters A, vol. 356, no. 2, pp. 131137, 2006.

[27] M. M. Rashidi, D. D. Ganji, and S. Dinarvand, "Explicit analytical solutions of the generalized Burger and Burger-Fisher equations by homotopy perturbation method," Numerical Methods for Partial Differential Equations, vol. 25, no. 2, pp. 409-417, 2009.

[28] H. Aminikhah and M. Hemmatnezhad, "An efficient method for quadratic Riccati differential equation," Communications in Nonlinear Science and Numerical Simulation, vol. 15, no. 4, pp. 835-839, 2010.

[29] S. H. Kachapi and D. D. Ganji, Nonlinear Equations: Analytical Methods and Applications, Springer, 2012.

[30] H. Jafari, A. M. Wazwaz, and C. M. Khalique, "Homotopy perturbation and variational iteration methods for solving fuzzy differential equations," Communications in Fractional Calculus, vol. 3, no. 1, pp. 38-48, 2012.

[31] Y. M. Qin and D. Q. Zeng, "Homotopy perturbation method for the q-diffusion equation with a source term," Communications in Fractional Calculus, vol. 3, no. 1, pp. 34-37, 2012.

[32] M. Javidi and M. A. Raji, "Combination of Laplace transform and homotopy perturbation method to solve the parabolic partial differential equations," Communications in Fractional Calculus, vol. 3, no. 1, pp. 10-19, 2012.

[33] J. S. Duan, R. Rach, D. Buleanu, and A. M. Wazwaz, "A review of the Adomian decomposition method and its applications to fractional differential equations," Communications in Fractional Calculus, vol. 3, no. 2, pp. 73-99, 2012.

[34] D. D. Ganji, "A semi-Analytical technique for non-linear settling particle equation of motion," Journal of Hydro-Environment Research, vol. 6, no. 4, pp. 323-327, 2012.

[35] J. Singh, D. Kumar, and Sushila, "Homotopy perturbation Sumudu transform method for nonlinear equations," Advances in Applied Mathematics and Mechanics, vol. 4, pp. 165-175, 2011.

[36] A. Ghorbani and J. Saberi-Nadjafi, "He’s homotopy perturbation method for calculating adomian polynomials," International Journal of Nonlinear Sciences and Numerical Simulation, vol. 8, no. 2, pp. 229-232, 2007.

[37] A. Ghorbani, "Beyond Adomian polynomials: he polynomials," Chaos, Solitons and Fractals, vol. 39, no. 3, pp. 1486-1492, 2009.

[38] S. Das and R. Kumar, "Approximate analytical solutions of fractional gas dynamic equations," Applied Mathematics and Computation, vol. 217, no. 24, pp. 9905-9915, 2011.
[39] G. K. Watugala, "Sumudu transform-a new integral transform to solve differential equations and control engineering problems," Mathematical Engineering in Industry, vol. 6, no. 4, pp. 319-329, 1998.

[40] S. Weerakoon, "Application of Sumudu transform to partial differential equations," International Journal of Mathematical Education in Science and Technology, vol. 25, no. 2, pp. 277-283, 1994.

[41] S. Weerakoon, "Complex inversion formula for Sumudu transform," International Journal of Mathematical Education in Science and Technology, vol. 29, no. 4, pp. 618-621, 1998.

[42] M. A. Aşiru, "Further properties of the Sumudu transform and its applications," International Journal of Mathematical Education in Science and Technology, vol. 33, no. 3, pp. 441-449, 2002.

[43] A. Kadem, "Solving the one-dimensional neutron transport equation using Chebyshev polynomials and the Sumudu transform," Analele Universitatii din Oradea, vol. 12, pp. 153-171, 2005.

[44] A. Kılıçman, H. Eltayeb, and K. A. M. Atan, "A note on the comparison between Laplace and Sumudu transforms," Iranian Mathematical Society, vol. 37, no. 1, pp. 131-141, 2011.

[45] A. Kılıçman and H. E. Gadain, "On the applications of Laplace and Sumudu transforms," Journal of the Franklin Institute, vol. 347, no. 5, pp. 848-862, 2010.

[46] H. Eltayeb, A. Kılıçman, and B. Fisher, "A new integral transform and associated distributions," Integral Transforms and Special Functions, vol. 21, no. 5-6, pp. 367-379, 2010.

[47] A. Kilıçman and H. Eltayeb, "A note on integral transforms and partial differential equations," Applied Mathematical Sciences, vol. 4, no. 1-4, pp. 109-118, 2010.

[48] A. Kılıçman, H. Eltayeb, and R. P. Agarwal, "On Sumudu transform and system of differential equations," Abstract and Applied Analysis, Article ID 598702, 11 pages, 2010.

[49] J. Zhang, "A Sumudu based algorithm for solving differential equations," Academy of Sciences of Moldova, vol. 15, no. 3, pp. 303-313, 2007.

[50] V. B. L. Chaurasia and J. Singh, "Application of Sumudu transform in Schödinger equation occurring in quantum mechanics," Applied Mathematical Sciences, vol. 4, no. 57-60, pp. 28432850, 2010.

[51] G. Adomian, Solving Frontier Problems of Physics: The Decomposition Method, vol. 60 of Fundamental Theories of Physics, Kluwer Academic, Dordrecht, The Netherlands, 1994.

[52] Z. Odibat and S. Momani, "Numerical methods for nonlinear partial differential equations of fractional order," Applied Mathematical Modelling, vol. 32, no. 1, pp. 28-39, 2008. 


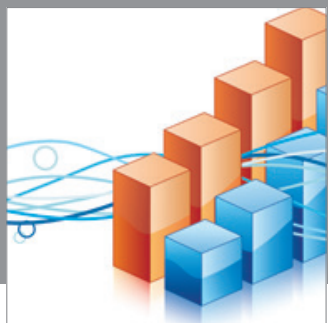

Advances in

Operations Research

mansans

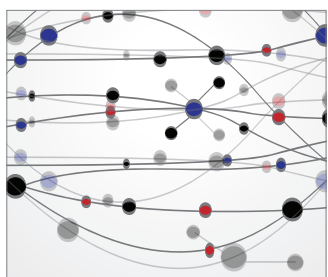

The Scientific World Journal
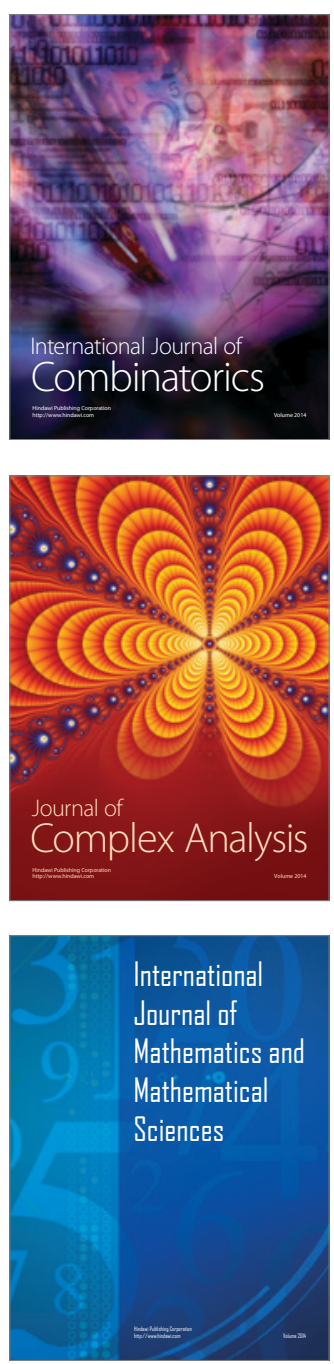
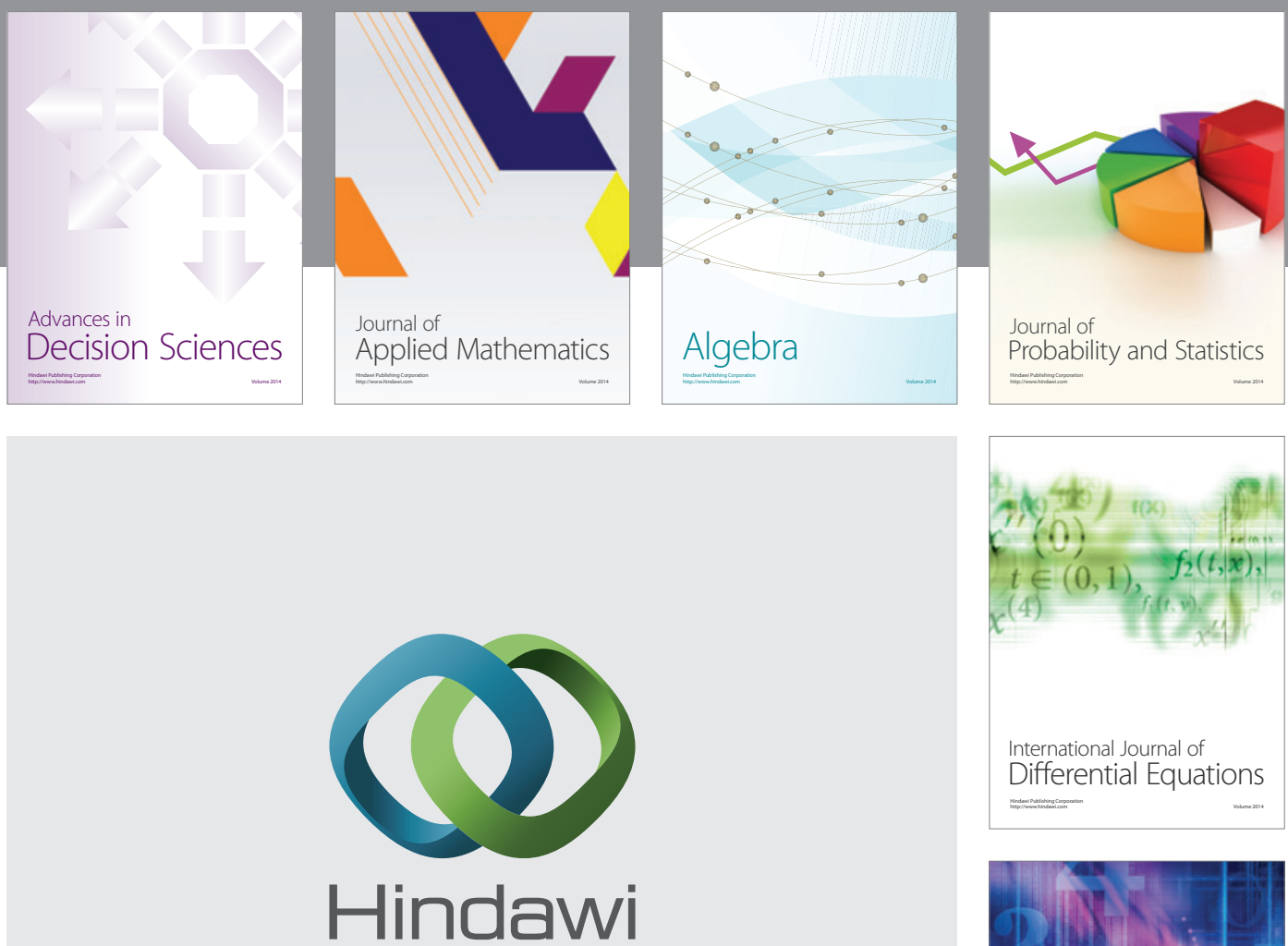

Submit your manuscripts at http://www.hindawi.com
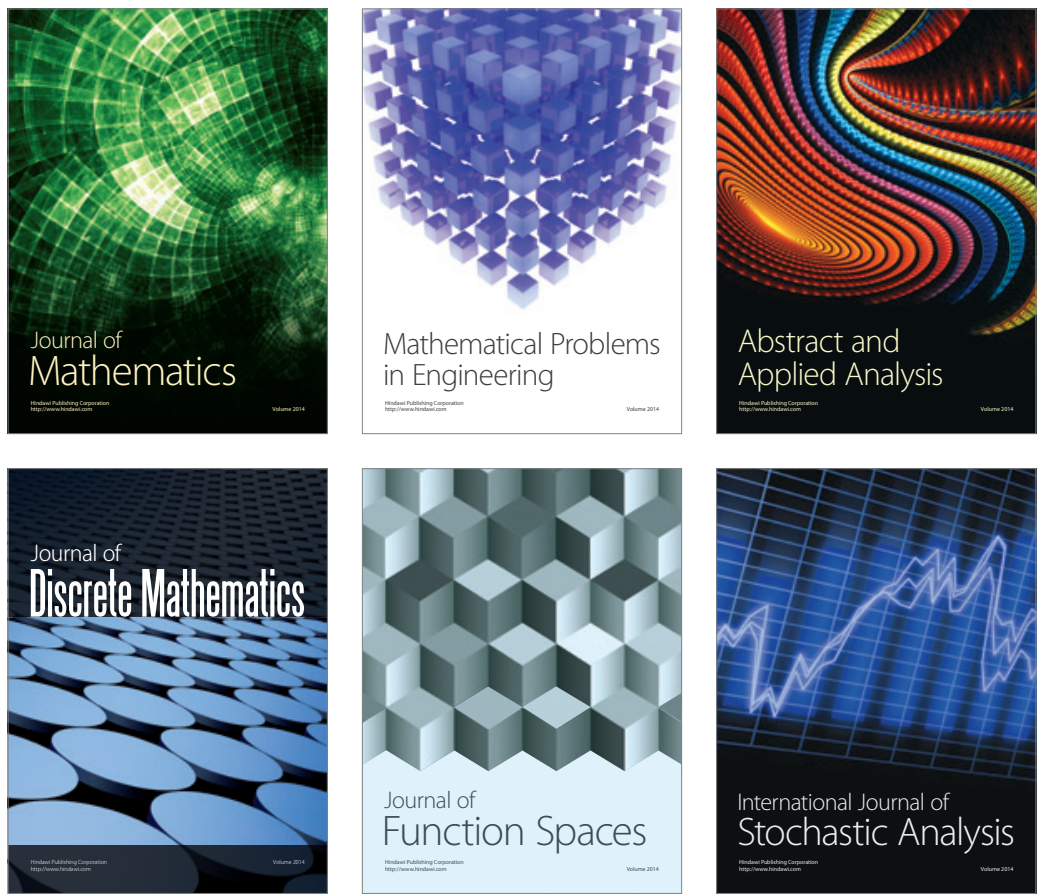

Journal of

Function Spaces

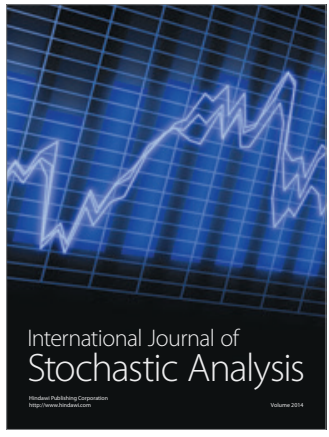

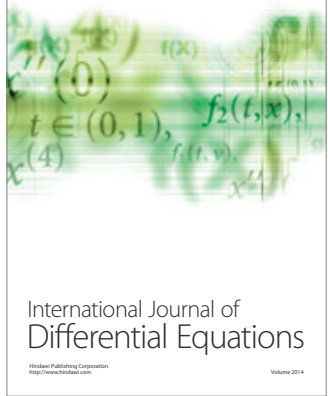
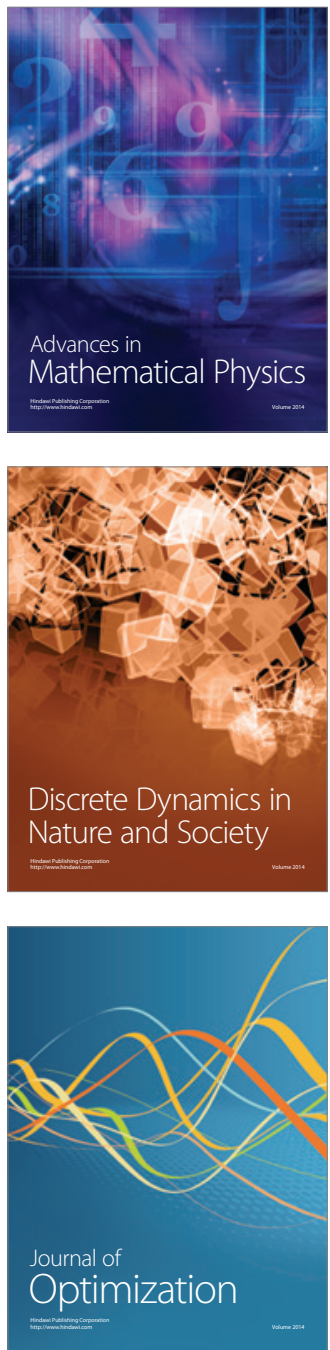